\title{
CALIBRAÇÃO DA METODOLOGIA NUMÉRICA DEM E APLICAÇÃO DO MODELO CALIBRADO EM UM CASO DE SCALE-UP EM UM TAMBOR ROTATÓRIO
}

\author{
D.A. SANTOS ${ }^{1 *}$, M.A.S. BARROZO ${ }^{1}$, C.R. DUARTE ${ }^{1}$, F. WEIGLER ${ }^{2}$, J. MELLMANN $^{2}$ \\ ${ }^{1}$ Universidade Federal de Uberlândia, Faculdade de Engenharia Química, Brasil \\ ${ }^{2}$ Instituto Leibniz de Engenharia Agrícola, Alemanha \\ "e-mail: dyrneq@yahoo.com.br
}

\begin{abstract}
RESUMO
Tambores rotatórios podem apresentar diferentes regimes de escoamento de partículas em seu interior, os quais estão diretamente relacionados às taxas de transferência de massa e energia e, consequentemente, à eficiência do processo em questão. Desta forma, foi realizada no presente trabalho uma investigação, tanto experimental quanto de simulação numérica, a respeito do escoamento granular no interior de um tambor rotatório sob diferentes condições operacionais. Para analisar o efeito da forma da partícula sobre o padrão de escoamento, foram utilizados grãos de arroz como material particulado. O Método dos Elementos Discretos (DEM) foi empregado em todas as simulações juntamente com a Técnica de Agrupamento (Clumping Technique), a qual permite a construção de partículas contendo formas arbitrárias. Para a calibração dos principais parâmetros presentes na modelagem $D E M$, um planejamento de experimentos foi utilizado. Perfis de velocidade de sólidos foram determinados experimentalmente, por meio de uma camera de alta velocidade, a fim de se validar o modelo em um caso de aumento de escala (scale up). Investigou-se, também, o efeito do comprimento do tambor sobre o padrão de escoamento granular. A calibração dos parâmetros da modelagem DEM através de um tambor em escala reduzida mostrou-se adequada para o emprego na previsão do escoamento em tambores rotatórios em uma escala superior, podendo, futuramente, ser empregada em uma escala industrial.
\end{abstract}

\section{INTRODUÇÃO}

O escoamento granular ou a dinâmica de partículas tem um papel importante em muitos processos industriais e uma melhor compreensão acerca de seu comportamento é essencial para questões de projeto e otimização.

Dentre outros equipamentos que envolvem escoamentos granulares, destaca-se o tambor rotatório, largamente utilizado em processos tais como, granulação (RAMACHANDRAN et al., 2008), mistura (MARIGO et al., 2012), moagem (METZGER e GLASSER, 2013), fermentação (LU et al., 2013), secagem (LOBATO et al., 2008; ARRUDA et al., 2009; FERNANDES et al., 2009; SILVA et al., 2012; SILVÉRIO et al., 2014) e recobrimento (SUZZI et al., 2012).

Apesar de possuir uma geometria relativamente simples, muitos estudos em tambores rotatórios tem se concentrado nas complexas interações partícula-partícula e partícula-parede no interior deste equipamento. Essas interações, a depender das condições operacionais e propriedades físicas dos materiais particulados, podem ocasionar o surgimento de diferentes regimes de escoamento (WATANABE, 1999; 
MELLMANN, 2001; LIU et al., 2005; DUBÉ et al., 2013).

Decisões de projetos e operacionais, na maioria dos processos desenvolvidos em tambores rotatórios, inclusive atualmente nas indústrias, são, rotineiramente, feitas sem uma compreensão fundamental dos fenômenos relacionados ao escoamento granular em seu interior.

Paralelamente aos estudos experimentais, as simulações numéricas surgem como uma ferramenta complementar na investigação dos escoamentos granulares com o intuito de superar as limitações impostas por questões de segurança nos processos e custo na confecção de equipamentos (MELLMANN et al., 2011; SANTOS et al., 2012; SANTOS et al., 2013; SANTOS et al., 2015).

As duas principais abordagens numéricas para o tratamento de escoamentos que envolvem partículas são a aproximação Euleriana e a aproximação Lagrangeana. $\mathrm{Na}$ abordagem Euleriana as fases são tratadas, independente de sua estrutura física, como interpenetrantes e contínuas, sendo as equações de transferência de massa, energia e quantidade de movimento, resolvidas sobre uma estrutura Euleriana fixa no domínio de cálculo de interesse.

Já na abordam Lagrangeana, cada partícula pertencente ao sistema é acompanhada, individualmente, com a consequente aplicação transiente do balanço de forças, levando em consideração as interações com as demais partículas e destas com os contornos fixos através de leis de contato (WEIGLER e MELLMANN, 2014; ALIZADEH et al., 2014).

Com base no exposto, este trabalho tem como objetivo a aplicação da metodologia Lagrangeana no escoamento granular em um tambor rotatório envolvendo partículas nãoesféricas (arroz). O estudo da metodologia Lagrangeana foi divido em duas partes: calibração do modelo através de um planejamento de experimentos e utilização do modelo calibrado em um caso de aumento de escala (scale-up).

\section{MÉTODOLOGIA}

\subsection{Metodologia Experimental}

No presente estudo, três diferentes configurações geométricas, utilizando-se de duas diferentes unidades de tambores rotatórios, uma delas permitindo a variação do comprimento do tambor, foram utilizadas (Tabela 1).

Tabela 1 - Diferentes configurações geométricas de tambores rotatórios utilizadas no presente trabalho.

\begin{tabular}{c|c|c}
\hline Conf. & Diâmetro (m) & Comprimento (m) \\
\hline D1 & 0,10 & 0,10 \\
D2 & 0,39 & 0,02 \\
D3 & 0,39 & 0,45 \\
\hline
\end{tabular}

Ambos os tambores rotatórios foram confeccionados em acrílico transparente e operados em regime de batelada. A fim de se evitar a ocorrência do escorregamento das partículas sobre a parede do tambor, uma lixa apropriada (P80) foi fixada sobre a parede interna ao longo dos tambores.

A calibração do modelo numérico foi realizada por meio da configuração de tambor rotatório D1 (Tabela 1), devido ao alto custo computacional característico deste tipo de modelagem. Para este propósito, os ângulos de repouso dinâmicos $(\theta)$, medidos experimentalmente através do software de tratamento de imagens ImageJ, foram comparados com os valores simulados sob as mesmas condições.

No que diz respeito à validação do modelo calibrado em um caso de aumento de escala (scale up), foram utilizadas as configurações D2 e D3 (Tabela 1) com o intuito de se analisar também o efeito do 
comprimento do tambor sobre a dinâmica de partículas.

Neste caso, os perfis de velocidade de partículas na metade da corda da superfície do leito de material (linha de referência mostrada na Figura 1), sob diferentes condições operacionais, foram medidos por meio de uma câmera de alta velocidade (até 2.000 quadros ou frames por segundo).

Figura 1 - Esquema do plano transversal do tambor utilizado na medida das velocidades.

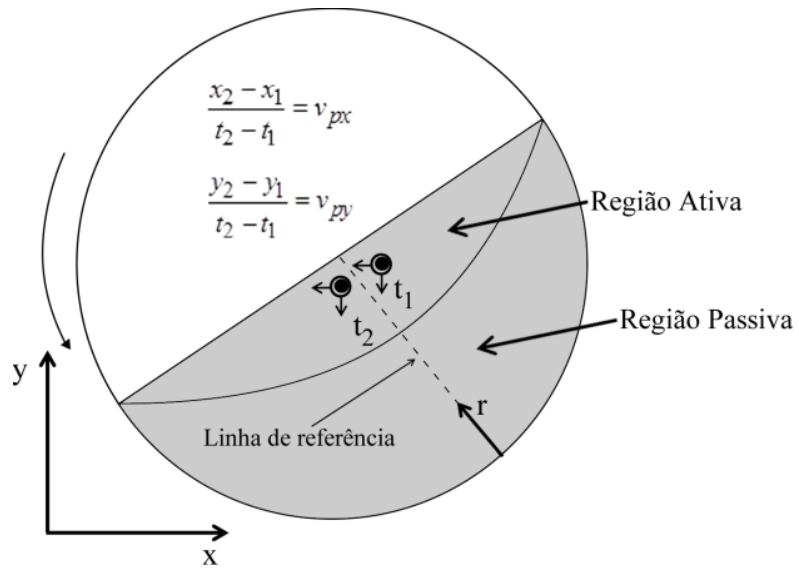

Esta técnica permite o rastreamento de partículas individuais em diferentes quadros ou frames e a velocidade é determinada através da subtração das posições da mesma partícula em dois frames distintos, imediatamente antes e depois da linha de referência, e a posterior divisão pelo intervalo de tempo entre os respectivos frames.

Como material particulado utilizou-se o arroz cujos valores medidos de densidade e porosidade do leito de material foram de 1465 $\mathrm{kg} / \mathrm{m}^{3}$ e 0,44 , respectivamente.

\subsection{Metodologia Numérica}

Todas as simulações Lagrangeanas (DEM) foram realizadas por meio do software comercial PFC3D ${ }^{\odot}$ (Particle Flow Code 3Dimensions). O software PFC3D ${ }^{\odot}$ é um código baseado no Método dos Elementos Discretos $(D E M)$, descrito com detalhes por Weigler e Mellmann (2014).
Para a calibração do modelo, foi realizada uma análise de sensibilidade paramétrica, por meio de um Planejamento Composto Central, a fim de se avaliar os efeitos do coeficiente de atrito $\left(\mu_{p}\right)$ e da razão de amortecimento $(\zeta)$, ambos variando de 0,149 a 0,701 , sobre o ângulo de repouso dinâmico do material $(\theta)$.

Em todas as simulações $D E M$ realizadas neste trabalho, um valor constante de coeficiente de mola de $2 \times 10^{5} \mathrm{~N} / \mathrm{m}$ foi empregado, o que tornou as simulações viáveis em termos de tempo computacional.

De acordo com as condições de contato locais e propriedades do material particulado, o software PFC $3 \mathrm{D}^{\odot}$ ajusta, automaticamente, o passo de tempo (times step) a fim de garantir uma estabilidade numérica. $\mathrm{O}$ passo de tempo para todas as simulações DEM variaram de $1 \times 10^{-6}$ a $1 \times 10^{-7} \mathrm{~s}$ e o tempo total de simulação foi de, aproximadamente, $20 \mathrm{~s}$.

Os grãos de arroz foram gerados através da metodologia de agrupamento (clump). Cada partícula de arroz foi criada utilizandose de cinco esferas sobrepostas, como visto na Figura 2.

Figura 2 - Partícula utilizada nas simulações DEM - (a) arroz; (b) clump ou representação computacional do arroz.

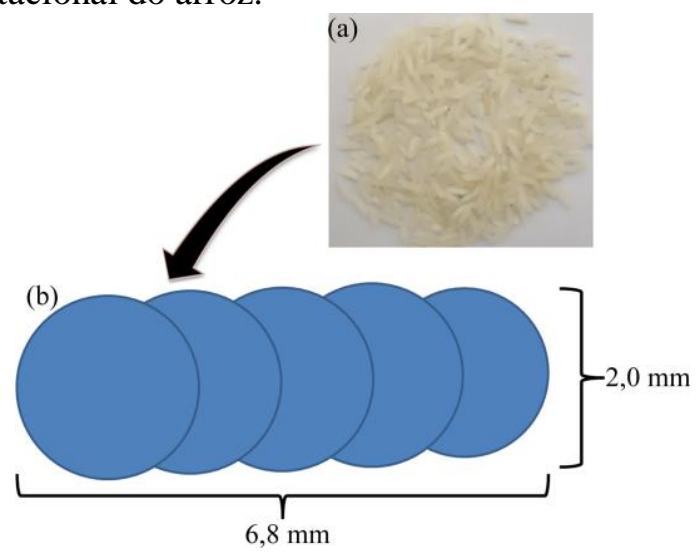

\section{RESULTADOS E DISCUSSÃO}

\subsection{Calibração do Modelo DEM}


Os valores das variáveis (níveis), coeficiente de atrito $\left(\mu_{p}\right)$ e razão de amortecimento $(\zeta)$ utilizados no Planejamento Composto Central, incluindo os valores medidos de ângulos de repouso dinâmico (resposta desejada), são dispostos, para o arroz, na Tabela 2.

Tabela 2 - Planejamento Composto Central utilizado na análise de sensibilidade dos principais parâmetros utilizados na modelagem DEM.

\begin{tabular}{c|c|c|c}
\hline \multirow{2}{*}{ Condição } & \multicolumn{2}{|c|}{ Fatores } & Ângulo de \\
\cline { 2 - 3 } & $\mu_{p}$ & $\zeta$ & repouso $\left(^{\circ}\right)$ \\
\hline 1 & 0,230 & 0,230 & $20,7-27,8$ \\
2 & 0,230 & 0,620 & 30,2 \\
3 & 0,620 & 0,230 & 38,1 \\
4 & 0,620 & 0,620 & 39,2 \\
5 & 0,149 & 0,425 & $17,2-22,8$ \\
6 & 0,701 & 0,425 & 39,23 \\
7 & 0,425 & 0,149 & 36,03 \\
8 & 0,425 & 0,701 & 37,3 \\
9 & 0,425 & 0,425 & 36,5 \\
\hline
\end{tabular}

Nota-se, a partir da Tabela 2 que, as simulações utilizando arroz nas condições $1 \mathrm{e}$ 5 revelaram um regime de caimento (slumping regime) do leito de material, como pode ser visto pela presença de dois diferentes ângulos de repouso dinâmico, o que não condiz com o observado experimentalmente sob as mesmas condições operacionais (regime de rolamento).

Neste regime, o material é carreado como um corpo sólido pela parede do tambor até alcançar o ângulo de repouso superior e então ocorre uma "avalanche" ou um caimento do material, formando, com a horizontal, um ângulo de repouso inferior.

Nota-se, também que, o ângulo de repouso dinâmico foi mais significativamente afetado pelo coeficiente de atrito $\left(\mu_{p}\right)$ do que pela razão de amortecimento $(\zeta)$. De um modo geral, os ângulos de repouso aumentaram tanto com o aumento do coeficiente de atrito $\left(\mu_{p}\right)$ quanto da razão de amortecimento $(\zeta)$.
Estes resultados numéricos mostraramse em concordância com aqueles observados na literatura (JUST et al., 2013).

Desta forma, altos valores de coeficiente de atrito e razão de amortecimento podem levar a uma significativa resistência aos movimentos de rotação e translação das partículas, providenciando, desta forma, altos valores de ângulos de repouso dinâmico em virtude do efetivo consumo de energia cinética.

O valor experimental de ângulo de repouso dinâmico para o arroz, sob as mesmas condições numéricas da Tabela 2 (grau de preenchimento de $15 \%$ e velocidade de rotação de $14,3 \mathrm{rpm}$ ), foi de $35,0^{\circ}$. Logo, o menor desvio dos dados experimentais foi obtido nas simulações cujos valores de coeficiente de atrito e razão de amortecimento foram, respectivamente, $\mu_{p}=0,425$ e $\zeta=$ 0,149 (condição 7 na Tabela 2), com um erro relativo de, aproximadamente, $2,9 \%$.

\subsection{Aplicação do modelo DEM Calibrado em um Caso de Scale-Up}

O objetivo principal desta seção é examinar a robustez do modelo DEM, calibrado através de um equipamento em pequena escala (configuração D1), através da aplicação em um caso de scale-up (configurações D2 e D3).

As Figuras 3-5 mostram uma análise do ângulo de repouso dinâmico através do plano transversal do tambor, tanto simulado quanto experimental, para diferentes condições operacionais. Com o intuito também, de analisar a influência do comprimento do tambor sobre a dinâmica das partículas, foram realizados experimentos com dois diferentes comprimentos: 0,02 m (configuração D2) e 0,45 m (configuração D3). 
Figura 3 - Plano transversal do tambor: grau de preenchimento de $10 \%$ e velocidade de rotação de 3,6 rpm (a) experimental (configuração D3); (b) simulado (configuração D2); (c) experimental (configuração D2).
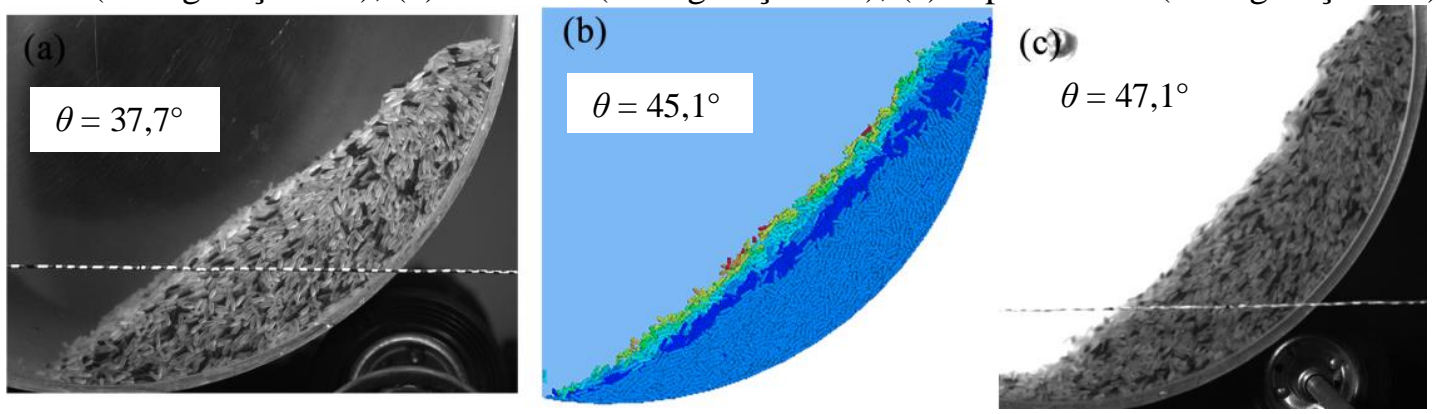

Figura 4 - Plano transversal do tambor: grau de preenchimento de $10 \%$ e velocidade de rotação de 6,2 rpm (a) experimental (configuração D3); (b) simulado (configuração D2); (c) experimental (configuração D2).
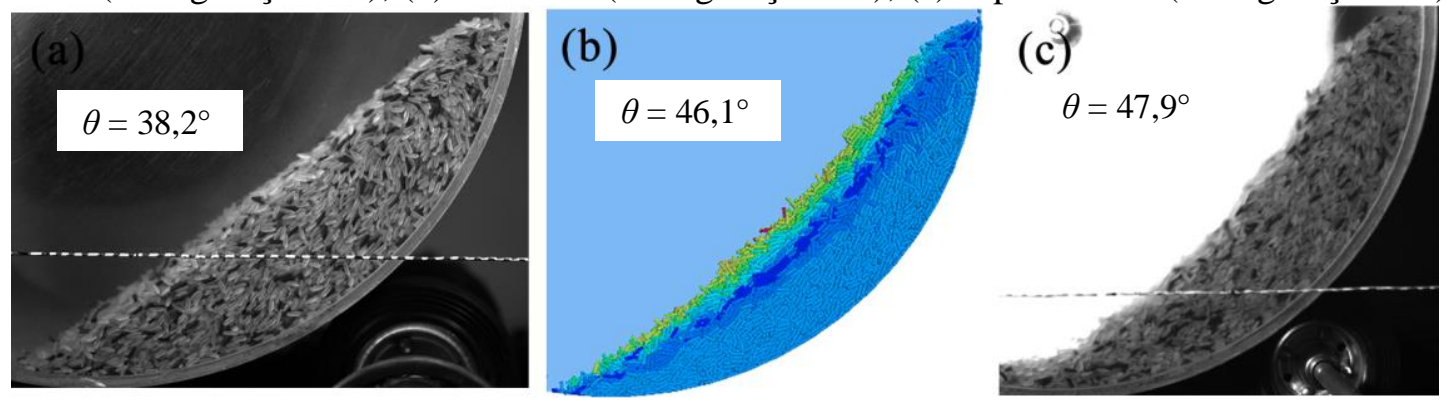

Figura 5 - Plano transversal do tambor: grau de preenchimento de 15\% e velocidade de rotação de 3,6 rpm (a) experimental (configuração D3); (b) simulado (configuração D2); (c) experimental (configuração D2).
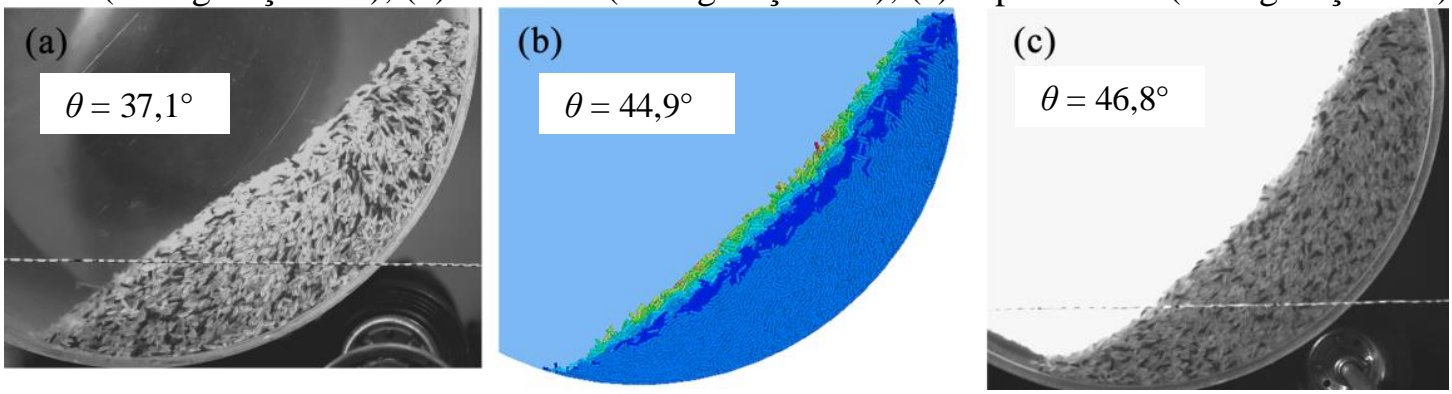

Observa-se, através das Figuras 3-5, quando se compara a inclinação do leito de partículas com relação à horizontal (ângulo de repouso dinâmico) nos experimentos realizados com diferentes comprimentos, uma considerável influência do comprimento do tambor sobre o padrão de escoamento granular.

A redução do comprimento do tambor ocasionou um aumento do ângulo de repouso do material, independente da condição operacional, devido à intensificação do efeito de parede o qual proporciona uma maior elevação do leito de material através do movimento ascendente das paredes laterais.

Os erros relativos, quando comparados os resultados simulados com os dados experimentais de ângulo de repouso utilizando o mesmo comprimento do tambor (configuração D2), foram, em média, de $4,0 \%$, enquanto que, os erros relativos quando comparados com a configuração D3 foram, em média, de 20,0\%.

Logo, nas simulações DEM do escoamento granular em um tambor rotatório, a simplificação da modelagem relacionada à 
diminuição da geometria do equipamento, quando comparada à escala real, pode levar a resultados que não condizem com a realidade.

No geral, o aumento da velocidade de rotação ocasionou um aumento no ângulo de repouso dinâmico do material, tanto para o simulado, quanto para o experimental, independente do comprimento do tambor. Por outro lado, o ângulo de repouso diminuiu com o aumento do grau de preenchimento do tambor (Figuras 3-5).
Para melhor avaliar a modelagem $D E M$ quando do aumento de escala, perfis de velocidade de sólidos medidos experimentalmente através de uma câmera de alta velocidade e utilizando dois diferentes comprimentos do tambor (configurações $D 2$ e D3), foram comparados com os resultados simulados sob as mesmas condições, como mostrados nas Figuras 6-8.

Figura 6 - Perfis de velocidade simulados e experimentais para o arroz sob diferentes configurações geométricas do tambor (configurações D2 e D3 da Tabela 1): grau de preenchimento de $10 \%$ e velocidade de rotação de 3,6 rpm - (a) velocidade resultante; (b) componente na direção x; (c) componente na direção y

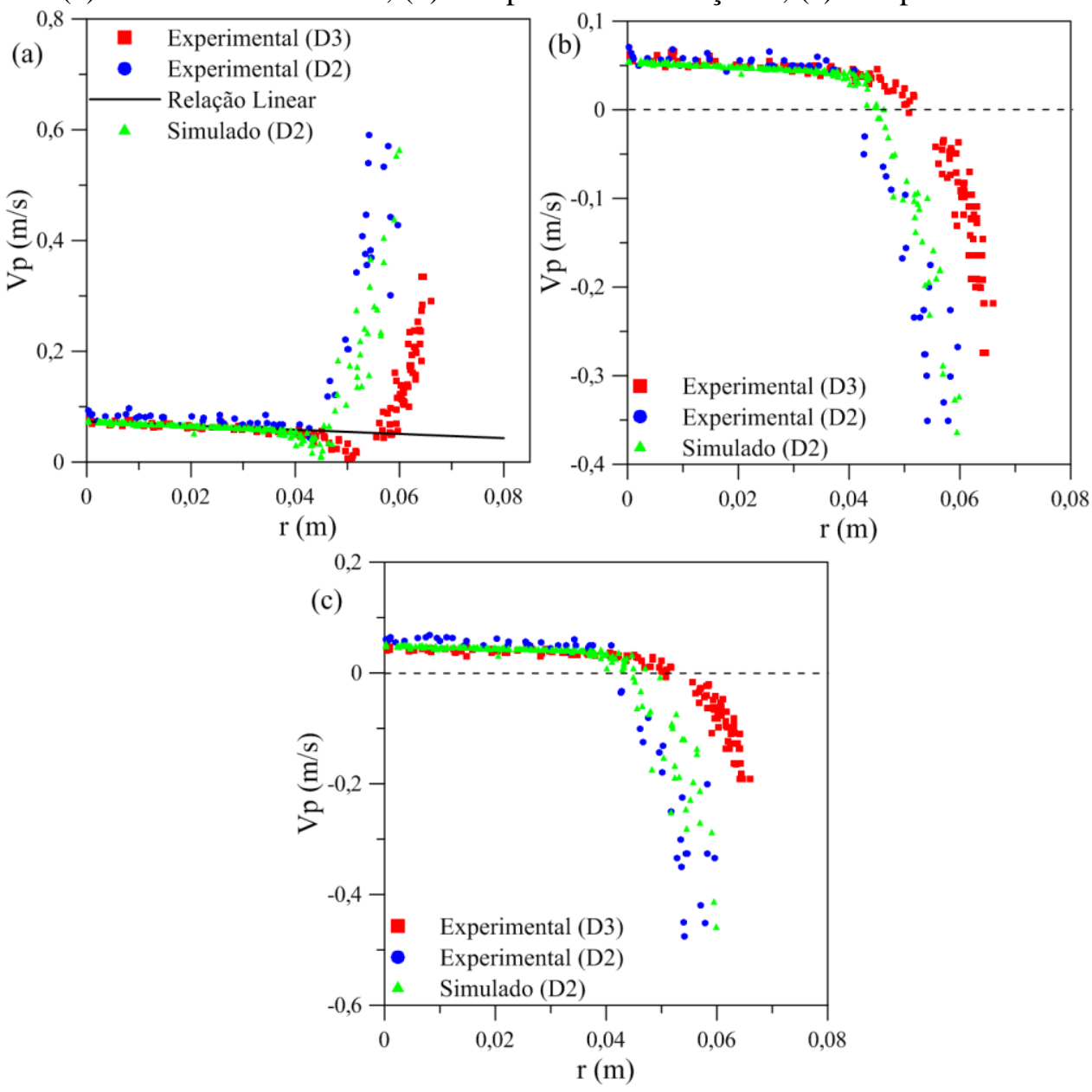

A relação linear, representada pela Equação 1, foi plotada nas Figuras 6-8 para auxiliar na comparação dos perfis de velocidade quando da utilização dos dois diferentes comprimentos e para indicar a transição entre as camadas ativa e passiva, tanto do experimental quanto do simulado.

$V_{\text {lin }}=\omega R$ 
sendo $V_{\text {lin }}, \omega$ e $R$ a velocidade linear, a velocidade angular e o raio do tambor, respectivamente.

Nota-se que, para ambos, experimental e simulado, os perfis de velocidade apresentaram características similares, ou seja, a velocidade de sólidos alcançou um valor máximo na superfície do leito, decresceu à medida que $r$ decresceu e tornouse, aproximadamente, nula na interface entre as regiões ativa e passiva onde ocorreu a inversão no sentido do escoamento pela ação da parede do tambor (presença de um ponto de inflexão) (Figuras 6-8).

Figura 7 - Perfis de velocidade simulados e experimentais para o arroz sob diferentes configurações geométricas do tambor (configurações $D 2$ e $D 3$ da Tabela 1): grau de preenchimento de $10 \%$ e velocidade de rotação de 6,2 rpm - (a) velocidade resultante; (b) componente na direção x; (c) componente na direção y

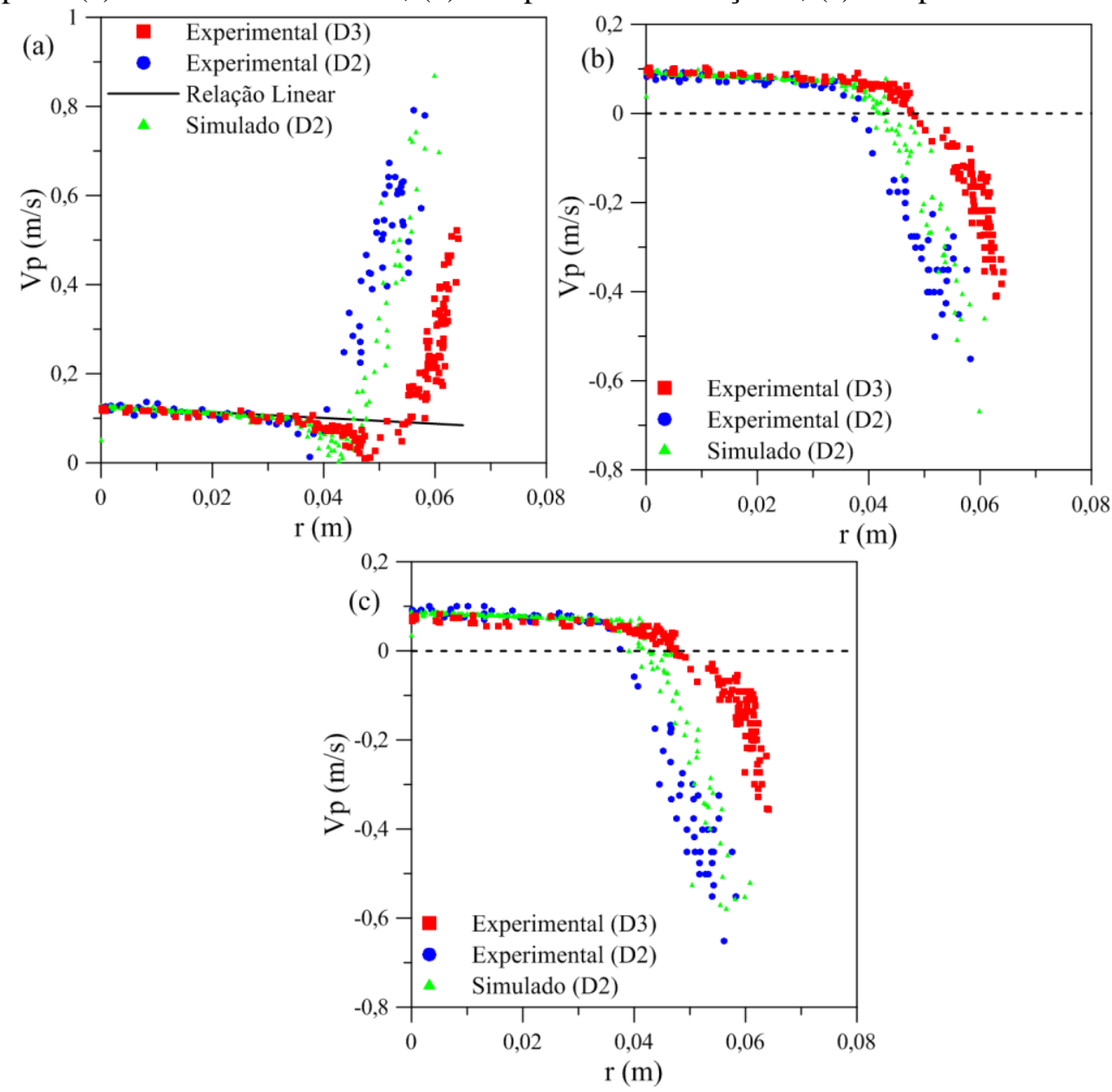

Duas diferentes regiões puderam ser visualizadas no plano transversal do tambor rotatório operando no regime de rolamento: uma região passiva, próxima à parede do tambor, onde as partículas movimentam-se mais lentamente, como um corpo sólido, somente devido à rotação do tambor (movimento difusivo), e uma região ativa, encontrada próxima à superfície do leito de material, caracterizada por um constante movimento convectivo possuindo, consequentemente, maiores valores de velocidade de partículas.

Em ambos os casos, simulado e experimental, independente do comprimento do tambor utilizado, as velocidades das 
partículas coincidiram com a relação linear próximo à parede do tambor, o que torna evidente que as partículas nesta região se movem como um corpo rígido, não possuindo, desta forma, velocidades relativas.

Logo, como era de se esperar, o comprimento do tambor possui influência somente sobre a região ativa, caracterizada por um movimento dinâmico descendente das partículas por ação, dentre outras, da força gravitacional.

Observa-se que a diminuição do comprimento do tambor causou um aumento, em todas as condições, na magnitude das velocidades das partículas na região ativa (Figuras 6-8).

Este fato está diretamente relacionado ao aumento do ângulo de repouso e a consequente reestruturação do leito de material (Figuras 3-5), visto que, uma maior elevação do material causa um aumento na energia potencial das partículas que, ao ser convertida em energia cinética pela força gravitacional, proporciona um aumento nas suas velocidades.

Figura 8 - Perfis de velocidade simulados e experimentais para o arroz sob diferentes configurações geométricas do tambor (D2 e D3 da Tabela 1): grau de preenchimento de 15\% e velocidade de rotação de 3,6 rpm - (a) velocidade resultante; (b) componente na direção x; (c) componente na direção y

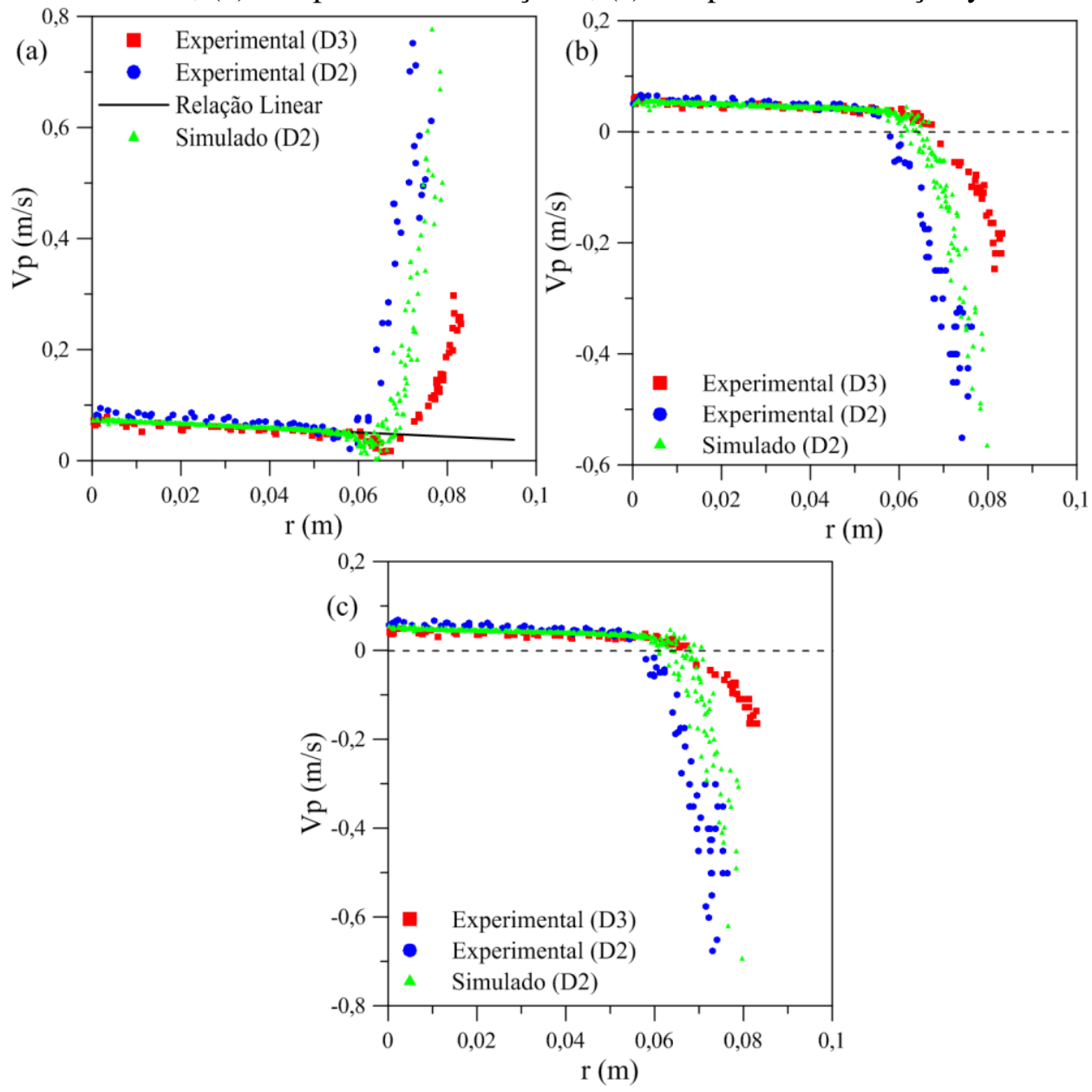

Nota-se uma satisfatória correspondência entre os perfis de velocidade simulados e experimentais para um comprimento de tambor de $2 \mathrm{~cm}$ ( configuração D2) para todas as condições operacionais. Um aumento na velocidade de 
rotação do tambor e/ou grau de preenchimento, causou um aumento na magnitude da velocidade das partículas na região ativa.

Desta forma, a calibração dos parâmetros da modelagem Lagrangeana, através de um tambor em escala reduzida, foi adequada para o emprego na previsão do escoamento do arroz em tambores rotatórios em uma escala superior, podendo, futuramente, ser empregada em uma escala industrial.

\section{CONCLUSÕES}

Foi possível a investigação do padrão de escoamento granular no interior de um tambor rotatório, utilizando partículas não-esféricas, através da abordagem Lagrangeana.

Notou-se uma grande influência do comprimento do tambor sobre o escoamento granular, sendo que, a redução do comprimento do tambor ocasionou um aumento do ângulo de repouso do material e um consequente aumento da magnitude da velocidade das partículas na região ativa.

A calibração dos parâmetros da modelagem Lagrangeana, através de um tambor em escala reduzida, foi adequada para o emprego na previsão do escoamento do arroz em tambores rotatórios em uma escala superior, podendo, futuramente, ser empregada em uma escala industrial.

\section{AGRADECIMENTOS}

Agradecemos o apoio financeiro concedido pela FAPEMIG e CAPES (Processo n ${ }^{\circ}$ 99999.001498/2014-02).

\section{REFERÊNCIAS}

ALIZADEH, E., BERTRAND, F., CHAOUKI, J. Discrete element simulation of particle mixing and segregation in atetrapodal blender. Comput. Chem. Eng., v. 64, p. 112, 2014.

ARRUDA, E.B., LOBATO, F.S., ASSIS, A.J., BARROZO, M.A.S. Modelling of fertilizer drying in roto-aerated and conventional rotary dryers. Drying Technol., v. 27, p. 1192-1198, 2009.

DUBÉ, O., ALIZADEH, E., CHAOUKI, J., BERTRAND, F. Dynamics of non-spherical particles in a rotating drum. Chem. Eng. Sci., v. 101, p. 486-502, 2013.

FERNANDES, N.J., ATAIDE, C.H., BARROZO, M.A.S. Modeling and experimental study of hydrodynamic and drying characteristics of an industrial rotary dryer. Braz. J. Chem. Eng., v. 26, p. 331341, 2009.

JUST, S., TOSCHKOFF, G., FUNKE, A., DJURIC, D., SCHARRER, G., KHINAST, J., KNOP, K., KLEINEBUDDE, P. Experimental Analysis of Tablet Properties for Discrete Element Modeling of an Active Coating Process. AAPS Pharm. Sci. Tech., v. 14, p. 402-411, 2013.

LIU, X. Y., SPECHT, E., MELLMANN, J. Experimental study of the lower and upper angles of repose of granular materials in rotating drums. Powder Technol., v. 154, p. 125-131, 2005.

LOBATO F.S., STEFFEN, V., ARRUDA, E.B., BARROZO, M.A.S. Estimation of drying parameters in rotary dryers using differential evolution. Journal of Physics Conference Series, v. 135, 012063, 2008.

LU, J., LI, D., CHEN, L., KITAMURA, Y., JIANG, W., LI, B. Simultaneous pretreatment and acidogenesis of solid food wastes by a rotational drum fermentation system with methanogenic leachate recirculation and 
andesite porphyry addition. Bioresour. Technol., v. 138, p. 101-108, 2013.

MARIGO, M., STITT, E.H. Discrete Element Method (DEM) for industrial applications: comments on calibration and validation for modeling of cylindrical pellets. Powder and Particle Journal, v. 32, p. 236-252, 2015.

MELLMANN, J. The transverse motion of solids in rotating cylinders-forms of motion and transition behavior. Powder Technol., v. 118, p. 251-270, 2001.

MELLMANN, J., IROBA, K.L., METZGER, T., TSOTSAS, E., MÉSZÁROS, C., FARKAS, I. Moisture content and residence time distributions in mixed-flow grain dryers. Biosystems Engineering, 109, 297-307, 2011.

METZGER, M. J., GLASSER, B. J. Simulation of the breakage of bonded agglomerates in a ball mill. Powder Technol., v. 237, p. 286-302, 2013.

RAMACHANDRAN, R., POON, J. M.-H., SANDERS, C. F.W., GLASER, T., IMMANUEL, C. D., DOYLE III, F. J., LITSTER, J. D., STEPANEK, F., WANG, F.Y., CAMERON, I. T. Experimental studies on distributions of granule size, binder content and porosity in batch drum granulation: Inferences on process modelling requirements and process sensitivities. Powder Technol., v. 188, p. 89-101, 2008.

SANTOS, D.A., ALVES, G.C., DUARTE, C.R., BARROZO, M.A.S. Disturbances in the hydrodynamic behavior of a spouted bed caused by an optical fiber probe: Experimental and CFD study. Ind. Eng. Chem. Res., v. 51, p. 3801-3810, 2012.

SANTOS, D.A., DADALTO, F.O., SCATENA, R., DUARTE, C.R., BARROZO,
M.A.S. A hydrodynamic analysis of a rotating drum operating in the rolling regime, Chem. Eng. Res. Des., v. 94, p. 204-2012, 2015.

SANTOS, D.A., PETRI, I.J., DUARTE, C.R., BARROZO, M.A.S. Experimental and CFD study of the hydrodynamic behavior in a rotating drum. Powder Technol., v. 250, p. 52-62, 2013.

SILVA, M. G., LIRA, T. S., ARRUDA, E. B., MURATA, V. V., BARROZO, M. A. S. Modelling of fertilizer drying in a rotary dryer: parametric sensitivity analysis. Braz. J. Chem. Eng., v. 29, p. 359-369.

SILVÉRIO， B. C., SANTOS, K. G., DUARTE, C. R., BARROZO, M. A. S. Effect of the friction, elastic, and restitution coefficients on the fluid dynamics behavior of a rotary dryer operating with fertilizer. Ind. Eng. Chem. Res., v. 53, p. 8920-8926, 2014.

SUZZI, D., TOSCHKOFF, G., RADL, S., MACHOLD, D., FRASER, S. D., GLASSER, B. J., KHINAST, J. G. DEM simulation of continuous tablet coating: Effects of tablet shape and fill level on inter-tablet coating variability. Chem. Eng. Sci., v. 69, p. 107121, 2012.

WATANABE, H. Critical rotation speed for ball-milling. Powder Technol., v. 104, p. 9599, 1999.

WEIGLER, F., MELLMANN, J. Investigation of grain mass flow in a mixed flow dryer. Particuology, v. 12, p. 33-39, 2014. 